\title{
Pulpotomía parcial con agregado de trióxido mineral (MTA) comparado con hidróxido de calcio en caries dentinaria profunda en pacientes con dientes permanentes inmaduros.
}

\section{Partial pulpotomy with mineral trioxide aggregate (MTA) versus calcium hydroxide in immature permanent teeth with deep caries}

\author{
Valentina Núñez Aravena ${ }^{1,2}$, Javiera Reyes Velásquez ${ }^{1,2}$, Andrea Cárdenas $^{1,2 *}$
}

\author{
1. Escuela de Odontología, Facultad de Medicina, \\ Pontificia Universidad Católica de Chile, Santiago, \\ Chile \\ 2. Proyecto Epistemonikos, Santiago, Chile \\ * Correspondencia Autor: Andrea Cárdenas \\ | Dirección: Centro Evidencia UC, Pontificia \\ Universidad Católica de Chile, Diagonal Paraguay \\ 476, Santiago, Chile | E-mail: acardenasd@uc.cl
}

\begin{abstract}
RESUMEN
Introducción: La pulpotomía parcial se utiliza para el tratamiento de caries con exposición pulpar en dientes permanentes inmaduros. El agregado de trióxido mineral (MTA) ha sido propuesto como uno de los biomateriales de elección para el tratamiento, pero existe incertidumbre en relación a su efectividad comparado con la del hidróxido de calcio. Métodos: Realizamos una búsqueda en Epistemonikos, la mayor base de datos de revisiones sistemáticas en salud, la cual es mantenida mediante el cribado de múltiples fuentes de información, incluyendo MEDLINE, EMBASE, Cochrane, entre otras. Extrajimos los datos desde las revisiones identificadas, analizamos los datos de los estudios primarios, realizamos un metanálisis y preparamos una tabla de resumen de los resultados utilizando el método GRADE. Resultados y conclusiones: Encontramos cinco revisiones sistemáticas, que incluyeron tres estudios primarios, de los cuales todos corresponden a ensayos aleatorizados. Concluimos que la pulpotomía parcial con agregado de trióxido mineral (MTA) podría resultar en poca o nula diferencia en la tasa de éxito comparado a la pulpotomía parcial con hidróxido de calcio, pero la certeza de la evidencia es baja.
\end{abstract}

\section{ABSTRACT}

Introduction: Partial pulpotomy is the treatment of choice following carious pulp exposure in immature permanent teeth. Mineral trioxide aggregate (MTA) has been suggested as the biomaterial first option for treatment, but there is still uncertainty regarding its effectiveness compared to calcium hydroxide. Methods: We searched in Epistemonikos, the largest database of systematic reviews in health, which is maintained by screening multiple information sources, including MEDLINE, EMBASE, Cochrane, among others. We extracted data from the systematic reviews, reanalyzed data of primary studies, conducted a meta-analysis and generated a summary of findings table using the GRADE approach. Results and conclusions: We identified five systematic reviews including three studies overall, of which all were randomized trials. We conclude that partial pulpotomy with mineral trioxide aggregate (MTA) may make little or no difference to success rate compared to partial pulpotomy with calcium hydroxide, however, the certainty of the evidence has been assessed as low. KEY WORD

Mineral trioxide aggregate; MTA; Calcium hydroxide; Partial pulpotomy; Immature permanent teeth; Carious pulp exposure; Epistemonikos; GRADE.

Int. J. Inter. Dent Vol. 13(3); 176 - 180, 2020. 


\section{PROBLEMA}

La caries dental no tratada en dientes permanentes es la enfermedad más prevalente en el mundo ${ }^{[1]}$. Las lesiones dentinarias profundas que no reciben un tratamiento oportuno pueden derivar en una exposición pulpar cariosa. Existen distintos tipos de tratamientos para aquellos casos que involucran dientes permanentes inmaduros, uno de los cuales es la pulpotomía parcial. Tradicionalmente, se ha usado el hidróxido de calcio como medicamento de elección para estimular la formación de dentina terciaria en este tratamiento. Sin embargo, se ha visto que presenta alta solubilidad en fluidos orales, baja resistencia mecánica y tendencia a la reabsorción ${ }^{[2]}$.

Frente a ello, se ha introducido a la práctica clínica el agregado de trióxido mineral (MTA) un material en base de silicato tricálcico biocompatible y bioactivo, que utiliza la humedad del medio oral para alcanzar sus propiedades mecánicas finales. Dentro de sus características destacan la formación de un puente dentinario más estable, una mejor capacidad de apexogénesis y sus propiedades desinfectantes ${ }^{[2]}$.

No obstante, este biomaterial tiene un alto costo y requiere de un mayor número de sesiones clínicas, por lo que su uso sigue siendo controvertido.

\section{MÉTODOS}

Realizamos una búsqueda en Epistemonikos, la mayor base de datos de revisiones sistemáticas en salud, la cual es mantenida mediante búsquedas en múltiples fuentes de información, incluyendo MEDLINE, EMBASE, Cochrane, entre otras. Extrajimos los datos desde las revisiones identificadas y analizamos los datos de los estudios primarios. Con esta información, generamos un resumen estructurado denominado FRISBEE (Friendly Summaries of Body of Evidence using Epistemonikos), siguiendo un formato preestablecido, que incluye mensajes clave, un resumen del conjunto de evidencia (presentado como matriz de evidencia en Epistemonikos), metanálisis del total de los estudios cuando sea posible, una tabla de resumen de resultados con el método GRADE y una sección de otras consideraciones para la toma de decisión.

\section{Mensajes clave}

- La pulpotomía parcial con agregado de trióxido mineral (MTA) podría resultar en poca o nula diferencia en la tasa de éxito comparado a la pulpotomía parcial con hidróxido de calcio (certeza de la evidencia baja).

- No se encontró evidencia evaluando el impacto de pulpotomía parcial con agregado de trióxido mineral (MTA) en cierre apical y dolor.

Acerca del conjunto de evidencia para esta pregunta

Cuál es la evidencia

Véase matriz de evidencia en Epistemonikos más abajo.

Qué tipo de pacientes incluyeron los estudios*

Qué tipo de desenlaces midieron
Encontramos cinco revisiones sistemáticas $^{[1], ~[2], ~[3], ~[4], ~[5], ~ q u e ~ i n c l u y e r o n ~}$ tres estudios primarios $[6],[7],[8]$, todos correspondientes a ensayos aleatorizados.

Todos los ensayos incluyeron pacientes con molares permanentes inmaduros con exposición pulpar cariosa.

Un ensayo también incluyó molares permanentes maduros, pero no fueron considerados para este resumen ${ }^{[7]}$

Solo un ensayo determinó el diagnóstico pulpar de sus dientes, siendo esta pulpitis irreversible ${ }^{[6]}$.

El promedio de edad de los participantes de los tres ensayos incluidos fue de 9,4 años (rango de 6 a 13,3 años) $)^{[6], ~[7], ~[8] . ~}$


pulpotomía parcial con agregado de trióxido mineral (MTA) contra pulpotomía parcial con hidróxido de calcio.

Los ensayos reportaron solo un desenlace, el cual fue presentado por las revisiones sistemáticas de la siguiente manera:

- Éxito clínico a los 6, 12, 24 y 36 meses

- Éxito radiográfico a los 6, 12, 24 y 36 meses

El seguimiento promedio de los ensayos fue de 14 meses con un rango que fluctúa entre 6 y 24 meses $^{[6],[7], ~[8]}$

* La información sobre los estudios primarios es extraída desde las revisiones sistemáticas identificadas, no directamente desde los estudios, a menos que se especifique lo contrario.

\section{RESUMEN DE LOS RESULTADOS}

La información sobre los efectos de la pulpotomía parcial con agregado de trióxido mineral (MTA) comparado con hidróxido de calcio en diente permanente inmaduro con caries dentinaria profunda está basada en tres ensayos aleatorizados ${ }^{[6],[7],[8]}$ que incluyen 228 dientes. Los tres ensayos midieron el desenlace tasa de éxito a 24 meses, el cual agrupa éxito clínico y radiográfico. Los desenlaces cierre apical y dolor no fueron reportados por la evidencia analizada.

El resumen de los resultados es el siguiente:

- La pulpotomía parcial con agregado de trióxido mineral (MTA) podría resultar en poca o nula diferencia en la tasa de éxito comparado a la pulpotomía parcial con hidróxido de calcio (certeza de la evidencia baja).

- No se encontró evidencia evaluando cierre apical.

- No se encontró evidencia evaluando dolor. 


\section{Pulpotomía parcial con MTA comparado con hidróxido de calcio en diente permanente inmaduro con caries dentinaria profunda}
Pacientes Intervención Comparación
Diente permanente inmaduro con caries dentinaria profunda Pulpotomía parcial con agregado de trióxido mineral (MTA)
Pulpotomía parcial con hidróxido de calcio

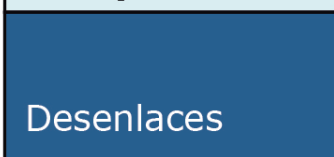

\begin{tabular}{|c|c|c|c|c|}
\hline \multirow[t]{2}{*}{ (1) } & nidroxido de call & 年 & \multirow{2}{*}{ (IC 95\%) } & \multirow{2}{*}{ (GRADE) } \\
\hline & \multicolumn{2}{|c|}{ Diferencia: dientes por 1000} & & \\
\hline \multirow[b]{2}{*}{ Tasa de éxito } & 839 por 1000 & 864 por 1000 & \multirow{2}{*}{$\begin{array}{l}\text { RR } 1,03 \\
(0,90 \text { a } \\
1,18)\end{array}$} & \multirow{2}{*}{$\begin{array}{c}\oplus \oplus \bigcirc \bigcirc^{1,2} \\
\text { Baja }\end{array}$} \\
\hline & \multicolumn{2}{|c|}{$\begin{array}{c}\text { Diferencia: } 25 \text { más } \\
\text { (Margen de error: } 84 \text { menos a } 151 \text { más) }\end{array}$} & & \\
\hline Cierre apical & \multicolumn{2}{|c|}{$\begin{array}{c}\text { El desenlace cierre apical no fue reportado por } \\
\text { la evidencia analizada. }\end{array}$} & -- & -- \\
\hline Dolor & \multicolumn{2}{|c|}{$\begin{array}{l}\text { El desenlace dolor no fue reportado por las } \\
\text { revisiones de manera individual, sino que } \\
\text { formó parte de los criterios utilizados para } \\
\text { evaluar el éxito clínico. }\end{array}$} & -- & -- \\
\hline
\end{tabular}

Margen de error: Intervalo de confianza del 95\% (IC 95\%).

RR: Riesgo relativo.

GRADE: Grados de evidencia del GRADE Working Group (ver más adelante).

* Los riesgos CON MTA están basados en los riesgos del grupo control en los estudios. El riesgo CON hidróxido de calcio (y su margen de error) está calculado a partir del efecto relativo (y su margen de error).

** La tasa de éxito se definió como la presencia de éxito clínico y radiográfico. Éxito clínico se define como la ausencia de manifestaciones clínicas: dolor espontáneo, dolor a la percusión o a la palpación y ausencia de necesidad de tratamiento de endodoncia. Éxito radiográfico se define como la reparación o resolución de lesiones periapicales y ausencia de necesidad de tratamiento de endodoncia.

${ }^{1}$ Se disminuyó un nivel de certeza de evidencia por riesgo de sesgo, debido a que se identificaron limitaciones asociadas al enmascaramiento de participantes y clínicos, sesgo de desgaste y ocultamiento de la secuencia de aleatorización.

${ }^{2}$ Se disminuyó un nivel de certeza de evidencia por imprecisión debido a que a cada extremo del intervalo de confianza se tomarían decisiones diferentes.

Siga el enlace para acceder a la versión interactiva de esta tabla (Interactive Summary of Findings - iSoF)

\begin{tabular}{|l|}
\hline Acerca de la certeza de la evidencia (GRADE)* \\
\hline$\oplus \oplus \oplus \oplus$ \\
Alta: La investigación entrega una muy buena indicación del efecto probable. La probabilidad de \\
que el efecto sea sustancialmente distinto† es baja. \\
$\oplus \oplus \oplus \bigcirc$ \\
Moderada: La investigación entrega una buena indicación del efecto probable. La probabilidad de \\
que el efecto sea sustancialmente distinto† es moderada. \\
$\oplus \oplus \bigcirc$ \\
Baja: La investigación entrega alguna indicación del efecto probable. Sin embargo, la probabilidad \\
de que el efecto sea sustancialmente distinto† es alta. \\
$\oplus \bigcirc \bigcirc$ \\
Muy baja: La investigación no entrega una estimación confiable del efecto probable. La \\
probabilidad de que el efecto sea sustancialmente distinto† es muy alta. \\
\hline *Esto es también denominado 'calidad de la evidencia' o 'confianza en los estimadores del efecto'. \\
†Sustancialmente distinto = una diferencia suficientemente grande como para afectar la decisión \\
\hline
\end{tabular}




\section{OTRAS CONSIDERACIONES PARA LA TOMA DE DECISIÓN}

\section{A quién se aplica y a quién no se aplica esta evidencia}

- Los resultados de este resumen son aplicables a todos los pacientes que tengan exposición pulpar cariosa en un diente permanente inmaduro, independiente del grupo dentario al que pertenezca (molares, premolares, caninos o incisivos permanentes inmaduros).

- No obstante lo anterior, no existirían razones clínicas para no aplicar los resultados a pacientes que tengan exposición pulpar por traumatismo en un diente permanente inmaduro.

\section{Sobre los desenlaces incluidos en este resumen}

- El desenlace "tasa de éxito" es crítico para la toma de decisión de acuerdo a la opinión de los autores de este resumen, lo que coincide con las revisiones sistemáticas identificadas.

- Si bien el desenlace cierre apical también es considerado crítico para la toma de decisiones, sólo una ${ }^{[1]}$ de las revisiones incluidas lo mencionó como un desenlace secundario a medir. Sin embargo, sus resultados no fueron reportados.

- El desenlace "dolor" que se considera crítico para estimar el éxito o fracaso de la intervención sólo fue evaluado como uno de los criterios de la tasa de éxito clínico en la mayoría de los ensayos.

\section{Balance daño/beneficio y certeza de la evidencia}

- La pulpotomía parcial con agregado de trióxido mineral (MTA) podría resultar en poca o nula diferencia en la tasa de éxito comparado a la pulpotomía parcial con hidróxido de calcio, pero la certeza de la evidencia ha sido evaluada como baja

- Considerando lo anterior y que adicionalmente no se encontraron revisiones que reportaran los desenlaces cierre apical y dolor, no es posible llevar a cabo un adecuado balance entre los riesgos y beneficios asociados al uso de la intervención.

\section{Consideraciones de recursos}

- Ninguna de las revisiones incluidas realizó un análisis de costoefectividad de la pulpotomía parcial realizada con MTA comparada con hidróxido de calcio.

- Dos revisiones sistemáticas [1], [3] concluyen que la decisión clínica debe considerar el costo-beneficio de cada biomaterial, dado que el uso de agregado de trióxido mineral (MTA) supone un mayor costo por el precio del biomaterial y la mayor cantidad de sesiones que requeriría por su alto tiempo de fraguado.

\section{Qué piensan los pacientes y sus tratantes}

- Debido a la similar tasa de éxito de estos biomateriales en la pulpotomía parcial por exposición cariosa en diente permanente inmaduro, se espera que los pacientes y sus tratantes se inclinen por el hidróxido de calcio, debido a su menor costo y menor número de sesiones clínicas en comparación al agregado de trióxido mineral (MTA).

- La pulpotomía con agregado de trióxido mineral (MTA) ha solucionado las desventajas del hidróxido de calcio, respecto a su alta solubilidad en fluidos orales, baja resistencia mecánica y tendencia a la reabsorción [1]. Sin embargo, esto ha significado mayor costo del tratamiento, junto a un posible incremento en el número de sesiones clínicas por su tiempo de fraguado y posibilidad de decoloración dentaria [1].

- Es importante que los pacientes tomen una decisión informada, conociendo las posibles ventajas y desventajas de los biomateriales, entendiendo que su rendimiento clínico en la pulpotomía es similar [1], [2], [3], [4], [5]

\section{Diferencias entre este resumen y otras fuentes}

- Las conclusiones de este resumen concuerdan con las cinco revisiones sistemáticas identificadas [1], [2], [3], [4], [5], las cuales reportan que la tasa de éxito de la pulpotomía parcial con MTA es similar a la del hidróxido de calcio, pudiendo utilizar ambos biomateriales con similar tasa de éxito. Además, declaran que no existe suficiente evidencia para hacer conclusiones de las ventajas de un material por sobre el otro.
- A su vez, las recomendaciones de la Guía de práctica clínica sobre terapia pulpar en dientes primarios y permanentes inmaduros de la American Academy of Pediatric Dentistry ${ }^{[9]}$, también indican la posibilidad de usar hidróxido de calcio o agregado de trióxido mineral (MTA) en la pulpotomía parcial para exposición cariosa en diente permanente inmaduro.

\section{¿Puede que cambie esta información en el futuro?}

- La probabilidad de que futuras investigaciones cambien las conclusiones de este resumen es alta, debido a la incertidumbre de la evidencia existente.

- Identificamos dos revisiones sistemáticas en curso [10], [11] en la International prospective register of systematic reviews (PROSPERO) que podrían arrojar datos relevantes sobre la tasa de éxito del MTA y el hidróxido de calcio en la pulpotomía parcial de dientes permanentes inmaduros.

- No identificamos ningún ensayo clínico aleatorizado en curso en el sitio web clinicaltrials.gov de la U.S. National Library of Medicine.

\section{CÓMO REALIZAMOS ESTE RESUMEN}

Mediante métodos automatizados y colaborativos recopilamos toda la evidencia relevante para la pregunta de interés y la presentamos en una matriz de evidencia.

Siga el enlace para acceder a la versión interactiva: Pulpotomía parcial con MTA comparado con hidróxido de calcio en diente permanente inmaduro con caries profunda.

\section{NOTAS}

Si con posterioridad a la publicación de este resumen se publican nuevas revisiones sistemáticas sobre este tema, en la parte superior de la matriz se mostrará un aviso de "nueva evidencia".

Este artículo es parte del proyecto síntesis de evidencia de Epistemonikos. Se elabora con una metodología preestablecida, siguiendo rigurosos estándares metodológicos y proceso de revisión por pares interno. Cada uno de estos artículos corresponde a un resumen, denominado FRISBEE (Friendly Summary of Body of Evidence using Epistemonikos), cuyo principal objetivo es sintetizar el conjunto de evidencia de una pregunta específica, en un formato amigable a los profesionales clínicos. Sus principales recursos se basan en la matriz de evidencia de Epistemonikos y análisis de resultados usando metodología GRADE. Mayores detalles de los métodos para elaborar este FRISBEE están descritos aquí (http://dx.doi.org/10.5867/medwave.2014.06.5997)

La Fundación Epistemonikos es una organización que busca acercar la información a quienes toman decisiones en salud, mediante el uso de tecnologías. Su principal desarrollo es la base de datos Epistemonikos (www.epistemonikos.org).

\section{DECLARACIÓN DE CONFLICTOS DE INTERESES}

Los autores declaran no tener conflictos de intereses con la materia de este artículo.

\section{AGRADECIMIENTOS}

Este resumen de evidencia fue elaborado con el apoyo metodológico del Centro Evidencia UC, Facultad de Medicina, Pontificia Universidad Católica de Chile. 


\section{Bibliografía}

1. Li Y, Sui B, Dahl C, Bergeron B, Shipman P, Niu L, Tay, F. Pulpotomy for carious pulp exposures in permanent teeth: a systematic review and meta-analysis. J Dent [Internet]. 2019 [cited $2020 \mathrm{Jul}$ 3]. Available from: https://linkinghub.elsevier.com/ retrieve/pii/S030057121930065X

2. Chen Y, Chen X, Zhang Y, Zhou F, Deng J, Zou J, Wang Y Materials for pulpotomy in immature permanent teeth: a systematic review and meta-analysis. BMC Oral Health [Internet]. 2019 [cited $2020 \mathrm{Jul}$ 3]. Available from: https://www.ncbi.nlm.nih.gov/pmc/ articles/pmid/31647004/

3. Elmsmari F, Ruiz X, Miró Q, Feijoo-Pato N, Durán-Sindreu F, Olivieri J. Outcome of Partial Pulpotomy in Cariously Exposed Posterior Permanent Teeth: A Systematic Review and Meta-analysis. J Endod. [Internet]. 2019 [cited 2020 Jul 3]. Available from: https://linkinghub.elsevier.com/retrieve/pii/S0099-2399(19)30512-6

4. Aguilar P, Linsuwanont P. Vital Pulp Therapy in Vital Permanent Teeth with Cariously Exposed Pulp: A Systematic Review. J Endod [Internet]. 2011 [cited 2020 Jul 3] Available from: https://www.jendodon.com/article/S0099-2399(10)01042-3/fulltext

5. Munir A, Zehnder M, Rechenberg D. Wound Lavage in Studies on Vital Pulp Therapy of Permanent Teeth with Carious Exposures: A Qualitative Systematic Review. J Clin Med [Internet]. 2020 [cited 2020 Jul 3]. Available from: https://www.ncbi.nlm.nih.gov/ pmc/articles/PMC7231275/

6. Chailertvanitkul P, Paphangkorakit J, Sooksantisakoonchai N, Pumas N, Pairojamornyoot W, Leela-Apiradee N. Randomized control trial comparing calcium hydroxide and mineral trioxide aggregate for partial pulpotomies in cariously exposed pulps of permanent molars. Int Endod J [Internet]. 2013 [cited 2020 Jul 3]. Available from: https://onlinelibrary.wiley.com/doi/abs/10.1111/iej.12225
7. Qudeimat M.A, Barrieshi-Nusair K.M, Owais A.I. Calcium Hydroxide vs. Mineral Trioxide Aggregates for Partial Pulpotomy of Permanent Molars with Deep Caries. Eur Arch Paediatr Dent [Internet]. 2007 [cited 2020 Jul 3]. Available from: https://link. springer.com/article/10.1007/BF03262577

8. Özgür B, Uysal S, Güngör HC. Partial Pulpotomy in Immature Permanent Molars After Carious Exposures Using Different Hemorrhage Control and Capping Materials. Pediatr Dent. [Internet]. 2017 [cited 2020 Jul 3]. Available from: .https://pubmed.ncbi. nlm.nih.gov/29070158/

9. Pulp Therapy for Primary and Immature Permanent Teeth [Internet]. AAPD. 2014 [cited $2020 \mathrm{Jul}$ 3]. Available from: https://www.aapd.org/media/Policies_Guidelines/ BP PulpTherapy.pdf

10. Kehua Que, Fang Wang, Zhouyan Pan, Jing Wen, Huaien Bu, Guangxu Sun. Comparison of mineral trioxide aggregate and calcium hydroxide of pulpotomy in immature permanent teeth: a systematic review and meta-analysis. PROSPERO [Internet]. 2019 [cited 2020 Jul 3] CRD42019125761 Available from: https://www.crd. york.ac.uk/prospero/display_record.php?ID=CRD42019125761

11. Prasanna Neelakantan, Venkateshbabu Nagendrababu, Shaju Jacob Pulikkotil, Jayakumar Jayaraman. Efficacy of materials used for pulpotomy in immature vita permanent teeth: a systematic review and network meta-analysis. PROSPERO [Internet]. 2018 [cited 2020 Jul 3] CRD42018084777 Available from: https://www.crd. york.ac.uk/prospero/display_record.php?ID=CRD42018084777 